\title{
HERA and the Asymptotic Behaviour of $F_{2}^{p}$
}

J R Forshaw and R G Roberts

January 1995 
DRAL is part of the Engineering and Physical Sciences Research Council

The Engineering and Physical Sciences Research Council does not accept any responsibility for loss or damage arising from the use of information contained in any of its reports or in any communication about its tests or investigations 
RAL-95-008

January 1995

\title{
HERA and the asymptotic behaviour of $F_{2}^{p}$
}

\author{
J.R. Forshaw and R.G. Roberts \\ Rutherford Appleton Laboratory, \\ Chilton, Didcot OX11 0QX, England.
}

\begin{abstract}
We examine the recent HERA data on the deep inelastic structure function, $F_{2}^{p}$. The data are analysed in terms of the 'double scaling' variables, $\sqrt{ }\left(\ln \frac{1}{x} \ln \ln Q^{2}\right)$ and $\sqrt{ }\left(\ln \frac{1}{x} / \ln \ln Q^{2}\right)$. Although the data can be well described by the 'double asymptotic scaling' prediction of perturbative QCD, we find that this is not the only possible scenario and hence conclude that, as yet, the HERA data is unable to identify the essential physics driving the small $x$ rise in $F_{2}^{p}$.
\end{abstract}



It has recently been argued [1] that the HERA data [2] on the deep inelastic structure function, $F_{2}^{p}$ support the perturbative QCD prediction of 'double asymptotic scaling' (DAS) $[3,4]$, with little or no need for a component of the type predicted by the formalism of BFKL (Balitsky, Fadin, Kuraev and Lipatov) [5]. Our purpose here is to demonstrate that DAS does not provide the only possible explanation of the data and that, so far, the HERA data cannot identify the underlying dynamics governing the small $x$ rise of $F_{2}^{p}$.

In the DAS approach of ref.[1] the 'double scaling' variables $\sigma \equiv \sqrt{ }\left(\ln \frac{x_{0}}{x} \ln \frac{t}{t_{0}}\right)$ and $\rho \equiv$ $\sqrt{ }\left(\ln \frac{x_{0}}{x} / \ln \frac{t}{t_{0}}\right)$ are introduced (where $\left.t=\ln Q^{2} / \Lambda^{2}\right)$. Perturbative QCD predicts, in the asymptotic limit of $\sigma, \rho \gg 1$ and for

$$
\lambda<\gamma / \rho
$$

that

$$
F_{2}^{p}(\sigma, \rho) \sim \exp \left[2 \gamma \sigma-\delta \sigma / \rho-\frac{1}{2} \ln \sigma-\ln \rho / \gamma\right] .
$$

Where $\gamma^{2}=4 N_{c} / \beta_{0}$ and $\delta=\left(1+2 n_{f} /\left(11 N_{c}^{3}\right)\right) /\left(1-2 n_{f} /\left(11 N_{c}\right)\right)$. The parameter $\lambda$ determines the small $x$ behaviour of the structure function, $F_{2} \sim x^{-\lambda}$, in the neighbourhood of $Q_{0}$. Note that for $\lambda=0, \sigma \gg 1, \sigma / \rho \sim 1$ the quantity

$$
\ln F_{2}^{p}(\sigma, \rho) / \sigma
$$

is independent of both $\rho$ and $\sigma$, i.e. 'double asymptotic scaling' ( $\rho$ scaling is ultimately violated if $\lambda \neq 0$ ).

In fig.(1), we compare the HERA data with the prediction of eq.(2). The data are plotted in different $\sigma$ bins as a function of $\rho$. We plot the ratio, $R$, of the data divided by the theoretical prediction. The overall normalisation of the theoretical prediction is considered free and fitted (separately) to the $\mathrm{H} 1$ and Zeus data. Taking $n_{f}=4$ and $N_{c}=3$ fixes $\gamma=1.2$ and $\delta=1.356$. The small- $x$ power is fixed by $\lambda$, and $\lambda=0$ for DAS ultimately to pertain. For $Q_{0}=1 \mathrm{GeV}$ and $x_{0}=0.1$ an excellent fit to the HERA data is found. In this fit, only the data for which $\sigma \geq 1, x \leq 0.01$ are included and the resulting $\chi^{2}$ is 50 for the 91 points satisfying these cuts. Nearly all these points lie in the upper two $\sigma$ bins of fig.(1) where the quality of the fit is clear.

However, we must be cautious in our interpretation of this result. The fact that the data are consistent with eq.(2) puts only a weak constraint upon the allowed range in $\lambda$. Since (for $x_{0}=0.1$ and $Q_{0}=1 \mathrm{GeV}$ ) all the HERA data lie at $\rho<3.5$ the implication would be that the behaviour of eq.(2) sufficiently far away from the boundary should hold for all $\lambda<0.34$. The only way to squeeze this limit on $\lambda$ is to get data at higher values of $\rho$. 
In addition, it is well known that DGLAP evolution $[4,6]$ from some starting distribution which is flat at small $x$ (i.e. $\lambda=0$ ) is unstable to evolution in $Q^{2}$, i.e. it rapidly develops a steep small $x$ growth. As a result, we should not be suprised to find that it is not possible to fit the HERA data with the DAS assumption that $\lambda=0$ for $Q_{0}$ much different from $1 \mathrm{GeV}$. This rapid growth of the small- $x$ gluon density in the vicinity of $Q_{0}$ is generated by the singular part of the gluon anomalous dimension arising from soft gluon emission.

Sensitivity to the singular part of the 1-loop anomalous dimension should be taken as a warning that higher-loop corrections are important. This has been demonstrated explicitly in ref.[7] where evolution from a starting distribution with $\lambda=0$ was shown to be sensitive to corrections at the 3- and 4-loop order (computed using the BFKL anomalous dimension [8] and the results on the quark anomalous dimensions of ref.[9]). This is as expected, since there are terms in the anomalous dimension matrix which are $\sim\left(\alpha_{s} / N\right)^{m}$ at the $m$-loop level (the small- $x$ limit is the small- $N$ limit, $N$ being the moment variable). These terms slow down the convergence of the DGLAP expansion at small $x$ and, when summed, reproduce the BFKL anomalous dimension. At small enough $x$ this summation will lead to the familiar power like behaviour of the gluon density, i.e. $x g \sim x^{-\lambda_{0}}$ where $\lambda_{0}=12 \ln 2 \alpha_{s} / \pi$. It has recently been argued that the region where the power behaviour is expected to dominate is outside that which is accessable at HERA [10] and that the summation merely affects the normalisation of the small $x$ distribution (i.e. the authors of ref.[10] support their earlier claims that DAS should pertain in the HERA region). We discuss the effects of including the full BFKL anomalous dimension in more detail in ref.[11]. In addition, the sub-leading $\sim \alpha_{s}\left(\alpha_{s} / N\right)^{m}$ contributions to the quark anomalous dimensions (calculated in [9]) have been shown to play an essential role [12]. Clearly it would be useful to also have the $\sim \alpha_{s}\left(\alpha_{s} / N\right)^{m}$ contributions to the gluon anomalous dimensions in order to allow more definite conclusions to be drawn. We have been discussing only the corrections to the anomalous dimensions but acknowledge that it is important to compute the coefficient function to the same accuracy. We recall that for a sufficiently large $\lambda$, there is no need to sum the anomalous dimensions since the leading behaviour is determined by the singular input (and not small $N$ ) and so is robust to higher order corrections. Of course, one is now no longer in a position to be able to discuss the dynamics responsible for generating the initial steepness.

Since an input which is flat at small $x$ for some value of $Q_{0}$ rapidly develops a steep behaviour, we might attempt to fit the data by approximating this growth by a non-zero value 
of $\lambda$ (e.g. as motivated by the BFKL summation) and a higher value of $Q_{0}$. In particular, we consider the opposite extreme to the DAS scenario, i.e. we take $Q_{0}=3 \mathrm{GeV}, x_{0}=1$, and $\lambda=0.38$ so that most of the HERA data no longer satisfies inequality (1). For $\sigma \gg 1$ and $\rho>\gamma / \lambda$, we then expect $[1,7,13]$

$$
F_{2}^{p}(\sigma, \rho) \sim \exp \left[\lambda \sigma \rho+\left(\gamma^{2} / \lambda-\delta\right) \sigma / \rho\right]
$$

The transition between eq.(2) and eq.(3) has been studied recently in ref.[13].

In fig.(2) we show the result of fitting eq.(3) to those HERA data which satisfy $\rho>\gamma / \lambda$. Again we use the canonical values for the QCD parameters and fit to the overall normalisation. For the 48 data points which satisfy the $\rho$ cut and have $\sigma>1$ and $x<0.01$ we find a value of $\chi^{2}=40$.

The quality of the fits using eq.(3) in its region of validity is quite acceptable and, in contrast to the fits using eq.(2), largely insensitive to the particular choices of $x_{0}$ and $Q_{0}$. Thus we are tentatively led to conclude that the claim of ref.[1], i.e. that a 'hard pomeron' is ruled out by the HERA data, are difficult to sustain.

In order to place our conclusion on a firmer footing, we perform the evolution of the parton densities using the full 2-loop evolution equations. Evolution using the summed (BFKL) anomalous dimension will be considered elsewhere [11]. In the limit of asymptotically large $\sigma$, we recover the results of eq.(2) and eq.(3). We parametrise the HERA data below $x=0.01$ by a simple power behaviour, i.e. $x^{-\lambda}$, whilst above $x=0.01$ we use a fit to that data fitted by the MRS group [14]. The normalisation of the small $x$ power behaviour is determined by ensuring continuity of the parton densities at $x=0.01$. This approach has the benefit that we can be sure our small $x$ behaviour is really a power law growth determined by $\lambda$, although it pays the price of having a discontinuity in the derivative of the parton densities at $x=0.01$.

In fig.(3), we perform evolution from $Q_{0}=3 \mathrm{GeV}$ with $\lambda=0.38 . \rho$ and $\sigma$ are defined with $x_{0}=1$ (this is an arbitrary choice since $x_{0}$ is not defined in the 2-loop evolution procedure). We find acceptable agreement to all of the HERA data for $Q^{2}>Q_{0}^{2}$, i.e. a $\chi^{2}$ per point of 160/138. This should be compared to fig.(2) whereupon one can see that the sub-asymptotic corrections play an important role in improving the quality of the fit in the region of $\rho<\gamma / \lambda$, as expected. We can further improve the quality of this type of fit by taking the exact power law behaviour at $Q_{0}$ only for $x<0.002$ for example, which ensures a smoother transition than the choice $x<0.01$. We then find a $\chi^{2}$ per point of $137 / 138$. 
In fig.(4) we consider the opposite scenario, corresponding to evolution from $Q_{0}=1 \mathrm{GeV}$ and $\lambda=0$ (see also ref.[17]). We take $x_{0}=0.1$ for comparison with fig.(1). Again the whole of the HERA data (with $x<x_{0}$ ) can be fitted well, i.e. a $\chi^{2}$ per point of 95/139. Improvement over fig.(1) is achieved in the low $\sigma$ regime, again as anticipated.

Not surprisingly, it is also possible to fit the HERA data by working in the scenario somewhat intermediate to the extreme scenarios just discussed. For example, evolution from $Q_{0}=2 \mathrm{GeV}$ with $\lambda=0.2$ is again able to obtain good agreement with the HERA data, i.e. $\chi^{2}=106$ for the 146 HERA data points. We have not discussed the possibility of different small- $x$ behaviours for the starting distributions of the gluons and sea-quarks but we expect such considerations to leave even more room to accommodate the dynamics of the high-energy (BFKL) summation.

Thus we have demonstrated that the HERA data on $F_{2}^{p}$ do not necessarily imply $\lambda=0$ with evolution driven by the asymptotic form of the 1-loop anomalous dimension.

\section{Acknowledgement}

We are grateful for discussion with Richard Ball, Keith Ellis, Stephano Forte and Robert Thorne.

\section{References}

[1] R.D.Ball and S.Forte, Phys. Lett. B335 (1994) 77; Phys. Lett. B336 (1994) 77.

[2] ZEUS collaboration: M. Derrick et al., DESY preprint 94-143 (1994);

H1 collaboration: J. Feltesse, V. Brisson and G. Rädel, Proc. 27th Int. Conf. on High Energy Physics, Glasgow, July 1994, eds. P.J. Bussey and I.G. Knowles.

[3] A.De Rujula et al., Phys. Rev. D10 (1974) 1649.

[4] Y.L.Dokshitzer, Sov. Phys. JETP 46 (1977) 641.

[5] E.A. Kuraev, L.N. Lipatov and V.S. Fadin, Sov. Phys. JETP 45 (1977) 199;

Ya. Ya. Balitsky and L.N. Lipatov, Sov. J. Nucl. Phys. 28 (1978) 822. 
[6] V.N. Gribov and L.N. Lipatov, Sov. J. Nucl. Phys. 15 (1972) 438;

L.N. Lipatov, Sov. J. Nucl. Phys. 20 (1974) 94;

G. Altarelli and G. Parisi, Nucl. Phys. B126 (1977) 298.

[7] R.K. Ellis, Z. Kunszt and E.M. Levin, Nucl.Phys. B420 (1994) 517.

[8] T.Jaroszewicz, Phys.Lett. B116 (1982) 291.

[9] S.Catani and F.Hautmann, Phys.Lett.B315 (1993) 157; Nucl. Phys. B427 (1994) 475.

[10] R.D.Ball and S.Forte, CERN-TH./95-1.

[11] J.R.Forshaw, R.G.Roberts and R.Thorne, in preparation.

[12] R.K. Ellis, F.Hautmann and B.R. Webber, Cavendish-HEP-94/18, Fermilab-PUB-95/006-T.

[13] N.N.Nikolaev and B.G.Zakharov, Phys.Lett. B327 (1994) 157.

[14] A.D. Martin, R.G. Roberts and W.J. Stirling, Phys. Rev. D50 (1994) 6734.

[15] M. Glück, E. Reya and A. Vogt, Phys. Lett. B306 (1993) 391.

[16] CTEQ collaboration: J. Botts et al., Phys. Lett. B304 (1993) 159; preprint MSU-HEP41024, CTEQ-404 Oct. 1994.

[17] R.D.Ball and S.Forte, CERN-TH.7422/94, to be published in the proceedings of 'QCD94', Montpellier, July 1994 (Nucl.Phys.B (Proc.Suppl.)).

\section{Figure Captions}

[1] The ratio $R=F_{2}$ (exp.)/ $F_{2}$ (theory) where $F_{2}$ (exp.) are the $\mathrm{H} 1$ data (solid circles) and Zeus data (open circles) of ref.[2] and $F_{2}$ (theory) is given by eq.(2), with free normalisation (separate for $\mathrm{H} 1$ and Zeus). Here $\sigma$ and $\rho$ are defined for $x_{0}=0.1, Q_{0}=1 \mathrm{GeV}$ and $\Lambda=230 \mathrm{MeV}$. Only data satisfying $\sigma \geq 1, x_{0} \leq 0.01$ (91 points) are used in the fit.

[2] The ratio $R=F_{2}$ (exp.)/ $F_{2}$ (theory) where $F_{2}$ (exp.) are the $\mathrm{H} 1$ data (solid circles) and Zeus data (open circles) of ref.[2] and $F_{2}$ (theory) is given by eq.(3), with $\lambda=0.38$, with free normalisation (separate for H1 and Zeus). Here $\sigma$ and $\rho$ are defined for $x_{0}=1, Q_{0}=3$ $\mathrm{GeV}$ and $\Lambda=230 \mathrm{MeV}$. Only data satisfying $\sigma \geq 1, x_{0} \leq 0.01$ and $\rho \geq \rho_{\text {crit }}=\gamma / \lambda$ (48 points) are used in the fit. The dashed line denotes the value of $\rho_{\text {crit }}$. 
[3] The ratio $R=F_{2}(\exp .) / F_{2}$ (theory) where $F_{2}$ (exp.) are the $\mathrm{H} 1$ data (solid circles) and Zeus data (open circles) of ref.[2] and $F_{2}$ (theory) is obtained by evolving, via the full 2-loop equations, an updated MRS(A) parametrisation for partons at $Q_{0}=3 \mathrm{GeV}$ where the gluon and sea-quark distributions are replaced by $A x^{-0.38}$ for $x \leq 0.01$ (see text). Here $\sigma$ and $\rho$ are defined for $x_{0}=1, Q_{0}=3 \mathrm{GeV}$ and $\Lambda=230 \mathrm{MeV}$.

[4] The ratio $R=F_{2}$ (exp.)/ $F_{2}$ (theory) where $F_{2}$ (exp.) are the H1 data (solid circles) and Zeus data (open circles) of ref.[2] and $F_{2}$ (theory) is obtained by evolving, via the full 2-loop equations, an updated MRS(A) parametrisation for partons at $Q_{0}=1 \mathrm{GeV}$ where the gluon and sea-quark distributions are replaced by a constant for $x \leq 0.01$. Here $\sigma$ and $\rho$ are defined for $x_{0}=0.1, Q_{0}=1 \mathrm{GeV}$ and $\Lambda=230 \mathrm{MeV}$. 

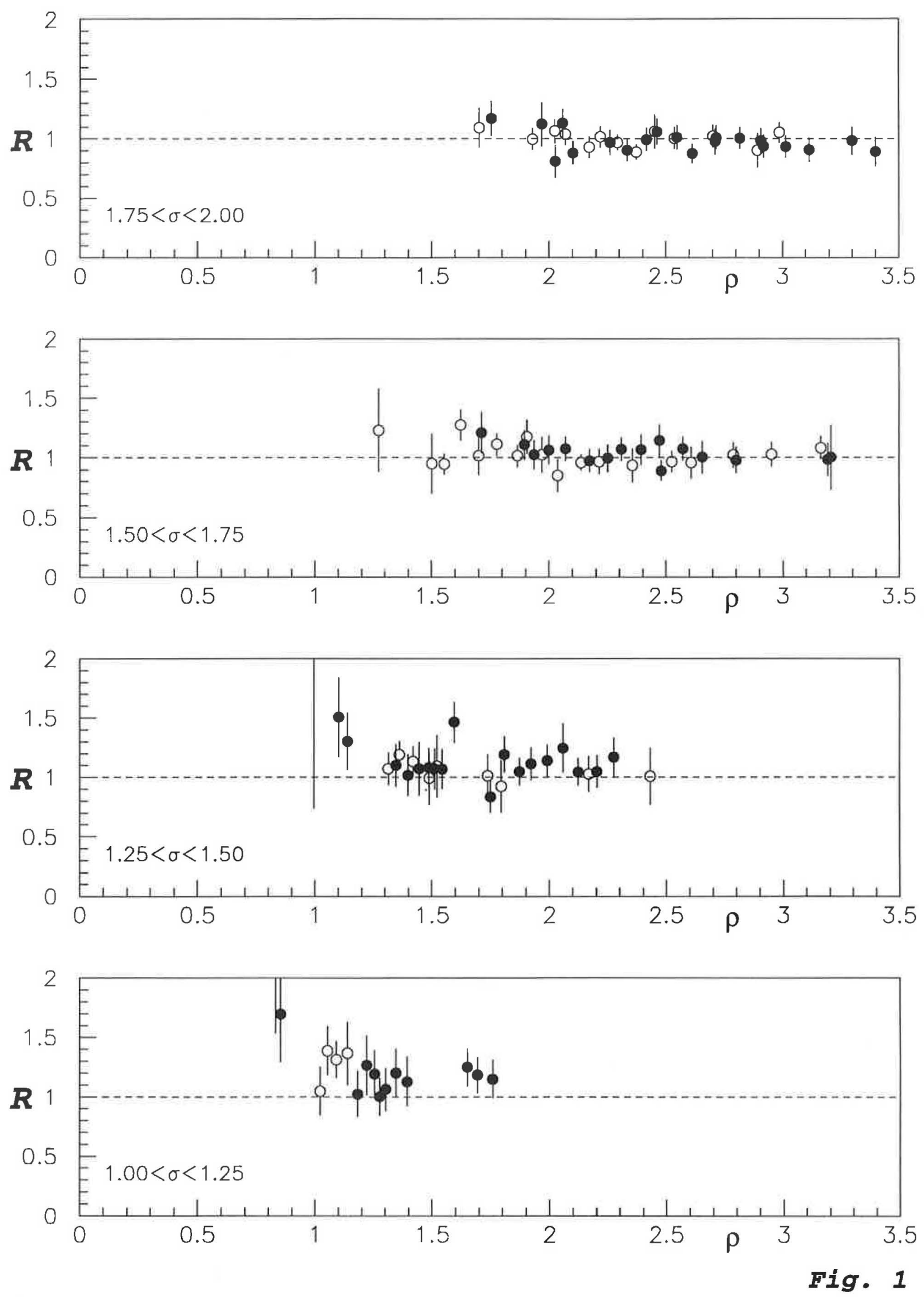

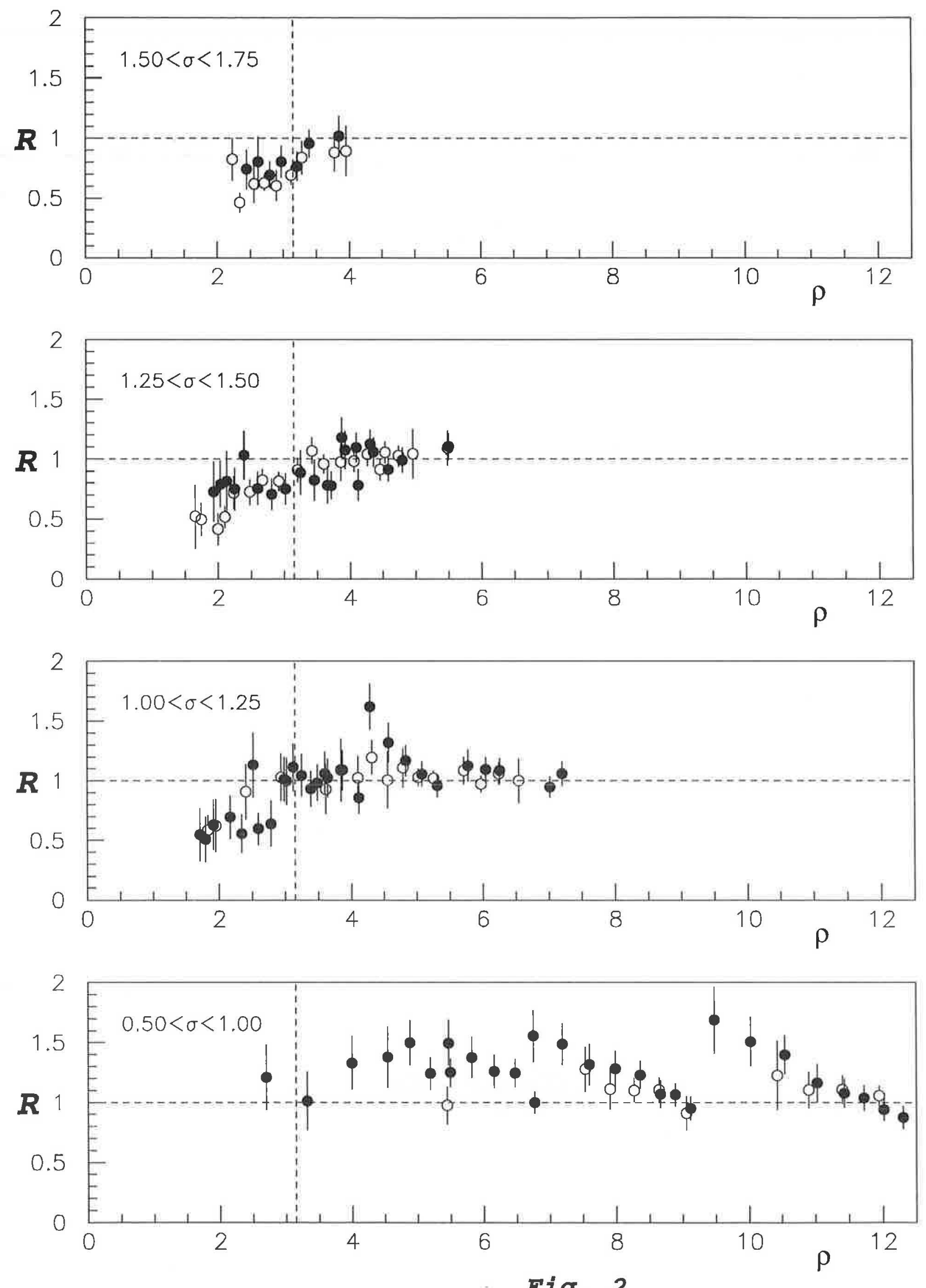

Fig. 2 

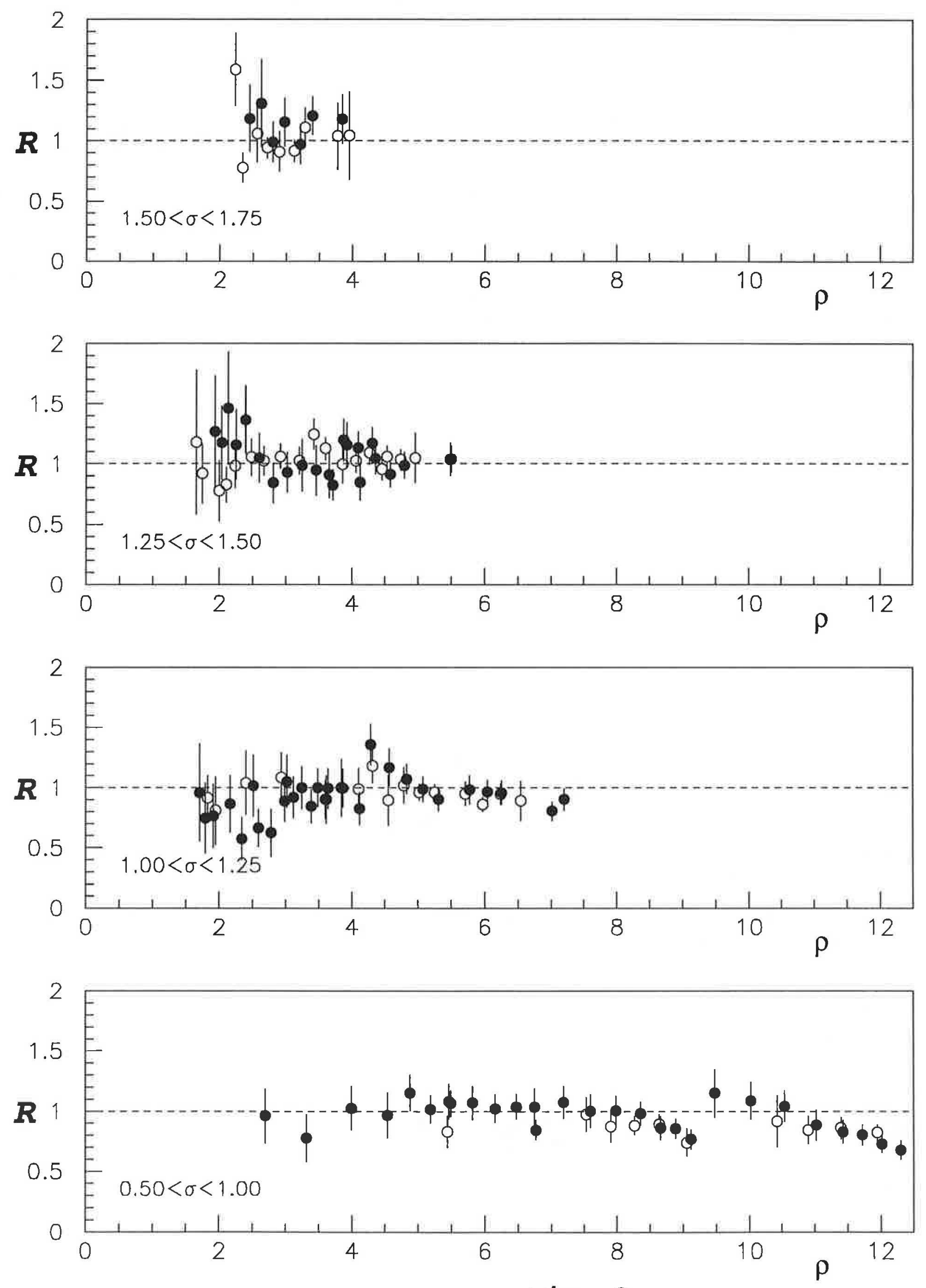

Fig. 3 

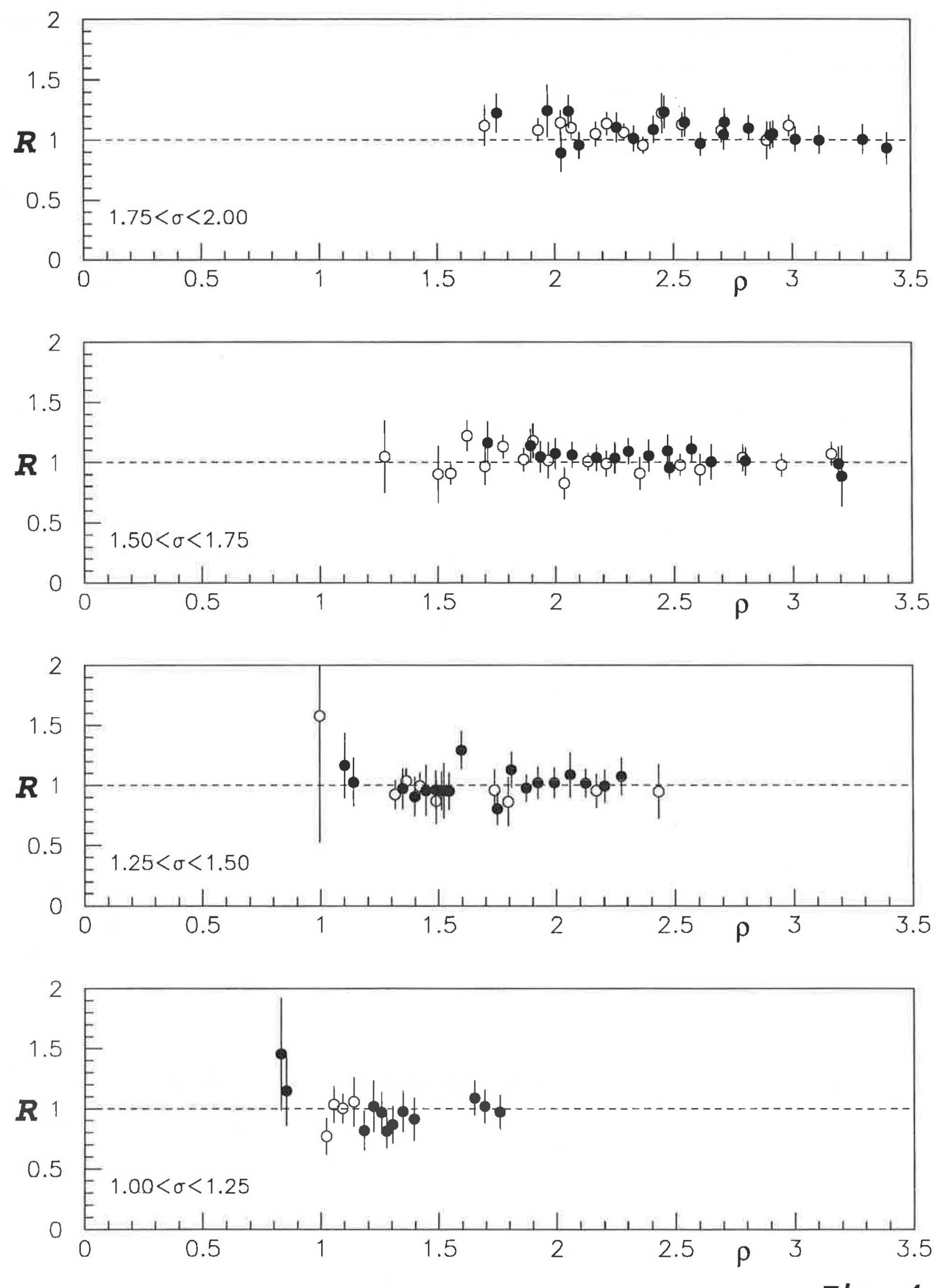

